\title{
Volumetric Reconstruction Technique for Multiple Parallel Circular Orbits CT
}

\author{
Jian $\mathrm{Fu}$ \\ School of Mechanical Engineering and Automation \\ Beijing University of Aeronautics and Astronautics \\ Beijing, 10091, China \\ e-mail: fujian706@buaa.edu.cn
}

\author{
Dekai Fan \\ School of Mechanical Engineering and Automation \\ Beijing University of Aeronautics and Astronautics \\ Beijing, 100191, China \\ e-mail: dkfan706@gmail.com
}

\begin{abstract}
Objective: The aim of this study is to develop a new volumetric reconstruction technique for cone beam computed tomography (CT) system with multiple parallel circular orbits, which can be used to suppress the cone-beam artifacts, permit the reconstruction of limited angle projections and decrease the noise. Methods: This technique includes two steps. Firstly the volume was reconstructed by the super-short-scan algorithm for each orbit. Then the multiple reconstructed volumes were weighted averaging in frequency domain to get the final results. In this step, the overlap situation of the imaging regions was analyzed and parameterized to implement the weighted averaging operation. Numerical simulations with the well-known FORBILD thorax phantom were executed to validate the proposed approach. Results: The slice images of the numerical phantom were reconstructed successfully. It demonstrates that the proposed method has the potential to realize the reconstruction of limited angle projections, decrease cone beam artifacts and improve the noise performance. Conclusion: This new method would be of interest for the development of new CT imaging devices.
\end{abstract}

Keywords-computed tomography; reconstruction algorithm; multiple parallel orbits; circular cone-beam orbit; super-shortscan

\section{INTRODUCTION}

$\mathrm{X}$-ray computed tomography (X-CT) enables the nondestructive observation of the internal structure of specimens and is a powerful analysis tool. It has become popular in medicine, biology and materials science since its introduction in the 1970s [1-3]. Over the last few decades, many novel CT techniques have been proposed. One of the recent developments is single circular orbit cone beam X-ray CT (CBCT), which is based on twodimensional area detector.

Compared with the conventional fan-beam X-CT, single circular orbit CBCT permits the production of hundreds of slices in parallel in a single gantry rotation and has a potential to achieve better scan efficiency and temporal resolution. However, it is impossible to avoid the cone beam artifacts because of data insufficiency [4, 5]. According to Tuy's condition [4], an exact 3D reconstruction from cone-beam projections is possible only when there exists at least one cone-beam source point on every plane that intersects the object. Unfortunately, single circular orbit CBCT does not satisfy this condition. The popular FDK algorithm developed by Feldkamp, Davis, and Kress [6] for single circular orbit CBCT is a generalization of 2D fan-beam filtered back-projection algorithm. It yields an exact image for the central plane and works well for small cone-beam angle. However, cone-beam artifacts will be produced in the slices away from the central plane and become serious as the angle increases. Although many variants of circular orbit CBCT have been proposed to implement the acquisition of a complete data set, they are at the cost of scan efficiency and temporal resolution. Recently the multiple parallel circular orbits cone beam X-CT technique [7] has been proposed to deal with the cone beam artifacts. It adopts several X-ray sources along the vertical direction and reduces the cone beam artifacts by decreasing the cone beam angle.

In this paper, a new volumetric reconstruction technique was proposed for cone beam computed tomography (CT) system with multiple parallel circular orbits, which can be used to suppress the cone-beam artifacts, permit the reconstruction of limited angle projections and decrease the noise. It firstly adopted the super-short-scan algorithm [8-10] to reconstruct the volume for each orbit of the multiple parallel orbits. Then the multiple reconstructed volumes were weighted averaging in frequency domain [7] to get the final results. The numerical simulation results using the well-known FORBILD thorax phantom validated the proposed approach. This new method would be of interest for the development of new CT imaging devices.

\section{SCANNING GEOMETRY}

Fig .1 depicts the scanning geometry of cone beam computed tomography system with multiple parallel circular orbits. In this geometry, each circular orbit is treated as the travel trajectory of one source point. At each view angle, the source emits cone-beam X-ray to penetrate the sample (here the human body) and the opposite area detector captures the projection image. Finally the algorithm reconstructed the sample volume from the recorded projection data set.

In Fig .1(a), the travel trajectories are discontinuous for limited angle CT. In this case, the conventional FDK algorithm can not work. We can adopt the classical supershort-scan algorithm to deal with this problem.

In Fig .1(c), there exist some overlap volumes. The overlap volume is the volume in the field of view (FOV) that can be reconstructed from multiple parallel cone-beam circular orbits. And we call the volume in the FOV that can only be reconstructed from one circular orbit conebeam non-overlap volume. One simple approach to deal with these overlap volumes is to reconstruct values at a particular location using only the data from the source that 
has the smallest cone-beam angle at that location. The image would have optimum accuracy but this is inefficient from a noise perspective. Another straightforward approach is to average the super-short-scan reconstructions from multiple parallel circular orbits. This is desirable for noise performance but would introduce cone-beam artifacts from the higher cone-beam angle data. As a compromise approach, the reconstructed volumes from different circular orbits can be combined using weighted averaging or ray-based weighting of the raw data that could be used, where the weights can be determined by the cone-beam angle of each orbit for the overlap volume .

\section{NEW RECONSTRUCTION TECHNIQUE}

Based on above analysis in Section II, we proposed a new reconstruction technique for multiple parallel circular orbits CT. Firstly the classical fan-beam super-short-scan algorithm is extended to cone-beam and applied to the projection data of each circular orbit to reconstruct the volume of interest (ROI). Then the overlap volumes are recognized and weighted. This technique adopts the supershort-scan algorithm to suppress the artifacts from the limited angle projections and deals with the overlap volume using weighted averaging.
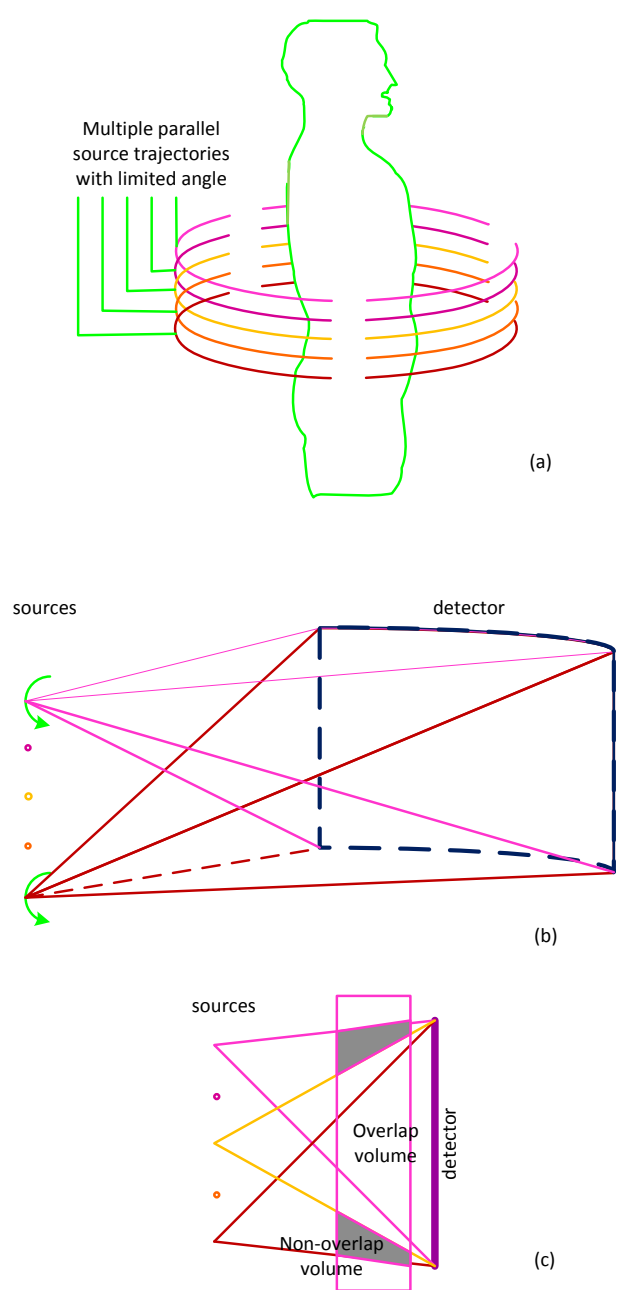

(c)

Figure 1. The scanning geometry of cone beam computed tomography system with multiple parallel circular orbits.
For a multiple parallel circular orbits CT with $m$ parallel orbits, the reconstruction procedure of the new algorithm is summarized as follows:

i) Perform 3D reconstruction of cone beam super-shortscan geometry for each orbit to obtain $m$ image volumes $f_{k}[\vec{x}](k=1,2, m) . \overrightarrow{\mathrm{x}}$ denotes any arbitrary point in the ROIs.

Here Noo's fan-beam reconstruction formula [9] was adopted and expressed by equations (1), (2) and (3). We extended these equations from fan-beam to cone-beam to match the scanning geometry in Fig .1 by using the wellknown cosine correction [6].

$$
\begin{aligned}
& f(\vec{x})=\frac{1}{2 \pi} \int_{\Lambda} d \lambda \frac{1}{\|\vec{x}-\vec{a}(\lambda)\|^{\prime}}\left[W(\lambda, \vec{n}) g_{\rho}(\lambda, \vec{n})\right]_{\vec{n}=\vec{n}(\lambda, \vec{x})} \\
& g_{F}(\lambda, \vec{n})=-\int_{S^{\prime}} d \vec{\alpha} h_{H}(\vec{n} \cdot \vec{\alpha}) g(\lambda, \vec{\alpha}) \\
& g^{\prime}(\lambda, \vec{\alpha})=\frac{\partial}{\partial \lambda} g(\lambda, \vec{\alpha})
\end{aligned}
$$

Here, $f$ denotes the reconstructed object, $\lambda$ the view angle, $a$ the position of the source, $W$ a weight used to account for information redundancy in the dataset, $n$ the unit vector orthogonal to the line connecting $\dot{a}$ and,$g_{F}$ the filtered projection with Hilbert filter, $g$ the measured fanbeam projection, $h_{H}$ Hilbert filter and $g$ ' the derivative of $g$ The detailed description about these equations can be found in [9].

ii) For each target axial cross-section $i$, analyze its overlap situation and extract the corresponding slices $f_{\mathrm{F}}(j=1,2, \ldots s)$ from $f_{\mathrm{ki}}(k=1,2, m)$. Here $s$ is smaller than $m$.

iii) Take FFT for the corresponding slices $f(j=1,2, \ldots s)$ to get $_{F_{\mathrm{ji}}}(j=1,2, \ldots s)$.

iv) Perform weighted averaging in frequency domain using formula (4).

$$
F_{\mathrm{wi}}=\sum_{\mathrm{j}=1}^{\mathrm{s}} \mathrm{W}_{\mathrm{ji}} F_{\mathrm{ji}}
$$

Here, $w_{\mathrm{ij}}$ is a weighting function to handle redundant data in frequency space. One can refer to [7] for details about this weighting function $\mathrm{w}_{\mathrm{ji}}$.

v) Take IFFT for $F_{\text {wi }}$ to obtain combined axial slice $f_{\text {wi }}$. vi)Steps ii)-v) are performed for all axial cross-sections. vii) All $f_{\mathrm{wi}}$ are stacked to produce the combined image volume $f_{\mathrm{w}}$.

\section{NUMERICAL SIMULATION}

Numerical simulations were executed in this section to investigate the validity and the performance of the proposed reconstruction algorithm. The system configuration parameters are shown in Table I. All the results are also compared to a reference system, which is a simple cone-beam axial scan with the same axial coverage and without any gaps using the standard FDK algorithm for reconstruction. The reference system is expected to have cone-beam artifacts due to the large cone-beam angle.

A numerical model based on the FORBILD thorax phantom was used in our simulation. The vertebrae of the thorax phantom are known to produce strong cone-beam artifacts at larger cone-beam angles. All simulations were monochromatic and conducted using analytic projection models to calculate the projection.

A noiseless simulation was firstly performed to display the reconstruction procedure of the proposed method and 
demonstrate its abilities of eliminating limited angle artifacts and cone-beam artifacts. Another simulation was then executed to investigate the noise property of the proposed method by adding 3\% Gaussian noise into the projections.

Fig .2 shows the reconstruction procedure of the proposed method with noiseless projection. Taking the reconstruction of $3 \mathrm{rd}$ cone-beam orbit as an example, panel I in figure 2 depicts the super-short-scan reconstruction. Fig .2(a) is the projection data set, Fig .2(b) the reconstructed axial slices and Fig .2(c) the 3D visualization of the images. Panel II shows the weighted average operation. Fig .2(d) displays the reconstruction results of all the five orbits. Fig .2(g) is 3D visualization of the final results after the weighted average operation in frequency domain. It has almost no disparity with the phantom and validates the proposed method.

Fig .3 demonstrates the performance of the proposed method on eliminating artifacts using the axial slice reconstructed with noiseless projections. Fig .3(a) shows the slice reconstructed from the reference system using the standard FDK algorithm. This slice is $50 \mathrm{~mm}$ away from the central plane and corresponds to half cone-beam angle about $4.8^{\circ}$. The cone-beam artifacts around the vertebra, marked by the red arrow in Fig .3(a), are quite visible. In contrast, there are no visible artifacts in the slice image reconstructed by the new algorithm displayed in Fig .3(b).

It is clear that the new reconstruction algorithm works well on eliminating cone-beam artifacts and permitting the reconstruction of limited angle projections. The gray curves in Fig .3 support quantitatively this conclusion.

Fig .4 repeats the demonstration in figure 3 but using the sagittal slice. Fig .4(a) and (b) show the slices reconstructed by the reference system using the standard FDK algorithm and by the multiple parallel circular orbits CT using the new method respectively. The images in Fig .4 verify again the validity of the new method on eliminating artifacts.

The application of the super-short-scan algorithm in the new method makes contribution to the reduction of limited angle artifacts. It avoids the estimation of the missing rays. The multiple parallel circular orbits architecture is responsible for the suppression of cone-beam artifacts.

Fig .4 repeats the demonstration in figure 3 but using the sagittal slice. Fig .4(a) and (b) show the slices reconstructed by the reference system using the standard FDK algorithm and by the multiple parallel circular orbits CT using the new method respectively. The images in Fig .4 verify again the validity of the new method on eliminating artifacts.

The application of the super-short-scan algorithm in the new method makes contribution to the reduction of limited angle artifacts. It avoids the estimation of the missing rays. The multiple parallel circular orbits architecture is responsible for the suppression of cone-beam artifacts.

Fig .4 repeats the demonstration in figure 3 but using the sagittal slice. Fig .4(a) and (b) show the slices reconstructed by the reference system using the standard FDK algorithm and by the multiple parallel circular orbits CT using the new method respectively. The images in Fig .4 verify again the validity of the new method on eliminating artifacts.
The application of the super-short-scan algorithm in the new method makes contribution to the reduction of limited angle artifacts. It avoids the estimation of the missing rays. The multiple parallel circular orbits architecture is responsible for the suppression of cone-beam artifacts.

TABLE I. SOME PARAMETERS OF THE SCANNING SYSTEM

\begin{tabular}{|c|c|}
\hline Scanning Parameters & Values \\
\hline Source to isocenter distance & $60.0 \mathrm{~cm}$ \\
\hline Detector to isocenter distance & $50.3 \mathrm{~cm}$ \\
\hline Axial or z-field of view & $16 \mathrm{~cm}$ \\
\hline Number of source arrays & 3 \\
\hline Number of source rows & 5 \\
\hline Spacing between source rows & $4 \mathrm{~cm}$ \\
\hline
\end{tabular}

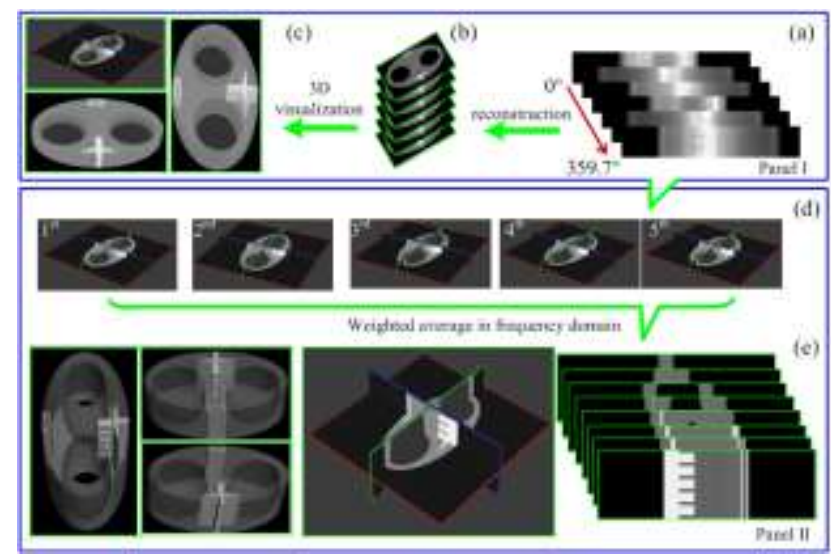

Figure 2. The recosntruction procedure of the proposed metnod.
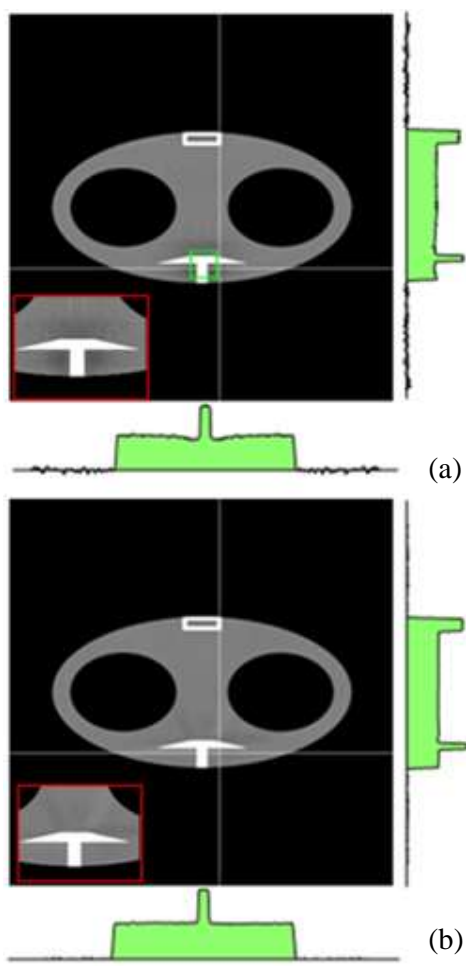

Figure 3. The typical axial slices reconstructed with noiseless projection. (a) is from the reference system using the standard FDK algorithm and (b) from mutiple parallel circurlar orbits CT using the new method. The inserts show the artifacts. 

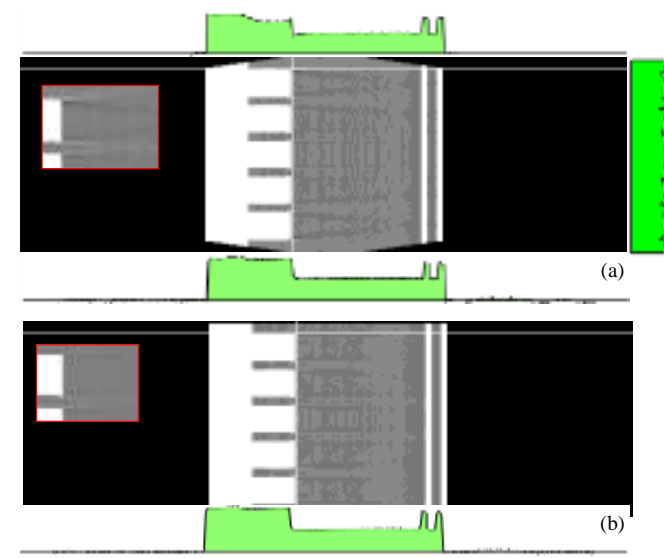

Figure 4. The typical sagittal slices reconstructed with noiseless projection. (a) is from the reference system using the standard FDK algorithm and (b) from mutiple parallel circurlar orbits CT using the new method. The inserts show the artifacts.

Fig .5 investigates the noise property of the proposed method using the axial slices reconstructed with noisy projections. Figures 4(a) and (b) correspond to the images reconstructed by the reference system using the standard FDK algorithm and by the multiple parallel circular orbits CT using the new method respectively. It seems that the new method has better noise performance than the reference system. The gray curves in Fig .5 support quantitatively this observation. The images in Fig .5 verify that the new method provides an improved noise performance.

This improvement on noise is attributed to the application of the weighted average operation in frequency domain and the super-short-scan algorithm.

\section{CONCLUSION}

In this paper, a new volumetric technique was proposed for multiple parallel circular orbits CT and validated by the numerical simulation study on FORBILD thorax phantom. Compared with the current methods, it was successful on eliminating artifacts, permitting the reconstruction of limited angle projection and improving the noise performance. The application of the super-short-scan algorithm contributes to the reduction on limited angle and cone-beam artifacts. The improvement on noise performance is attributed to the weighted average operation on 3D volumes reconstructed by the super-shortscan algorithm.

\section{ACKNOWLEDGMENT}

This work was partially supported by the large scale science facilities joint fund by National Natural Science Foundation of China and Chinese Academy of Science (11179009,U1432101), China Beijing Natural Science Foundation (7152088), Program for New Century Excellent Talents in University (NCET) from Ministry of Education of P. R. China and Beijing NOVA program (2009A09).
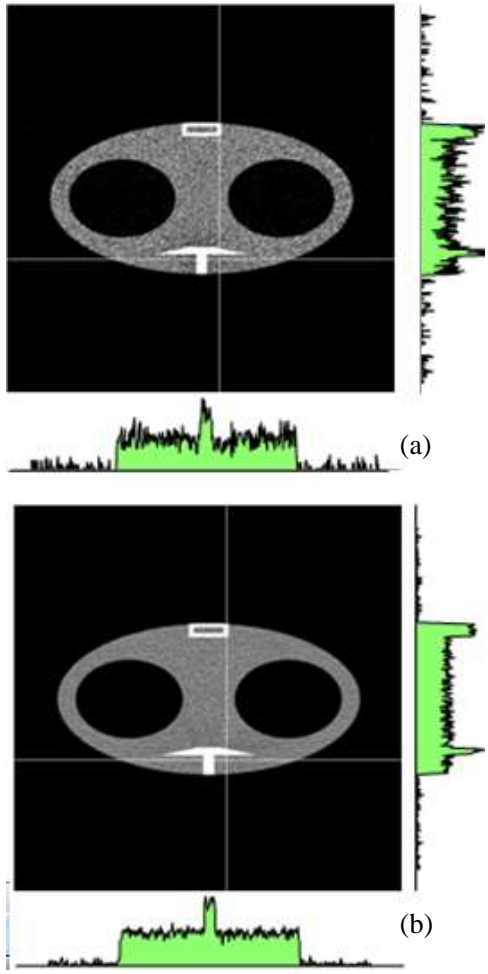

Figure 5. The typical axial slices reconstructed with noisy. (a) is from the reference system using the standard FDK algorithm and (b) from mutiple parallel circurlar orbits CT using the new method.

\section{REFERENCES}

[1] A. C. Kak and M. Slaney, Principles of Computerized Tomographic Imaging. New York: IEEE Press, 1988.

[2] J. Hsieh, Computed Tomography: Principles, Design, Artifacts, and Recent Advances. Bellingham, WA: SPIE, 2003

[3] J. Fu, H. Lu, B. Li, L. Zhang and J. Sun, "X-CT imaging method for large objects using double offset scan mode," Nuclear Instruments and Methods in Physics Research Section A: Accelerators, Spectrometers, Detectors and Associated Equipment, vol. 575, pp. 519-523, 2007

[4] H. K. Tuy, "An Inversion Formula for Cone-beam Reconstruction," SIAM Journal on Applied Mathematics, vol. 43 , pp. 546-552, 1983.

[5] B. D. Smith, "Cone-beam Tomography: Recent Advances and a Tutorial Review,” Optical Engineering, vol. 29, pp. 524-534, 1990.

[6] L. A. Feldkamp, L. C. Davis, J. W. Kress, "Practical Cone-beam Algorithm," Journal of the Optical Society of America, vol. 1, pp. 612-619, 1984.

[7] J. Baek and N. J. Pelc, "A New Method to Combine 3D Reconstruction Volumes for Multiple Parallel Circular Cone Beam Orbits," Medical physics, vol. 37, pp. 5351-5360, 2010.

[8] D. L. Parker, "Optimal Short Scan Convolution Reconstruction for Fan Beam CT," Medical Physics, vol. 9, pp. 254-257, 1982.

[9] F. Noo, M. Defrise, R. Clackdoyle R et al., "Image Reconstruction from Fan-beam Projections on Less Than a Short Scan," Physics in Medicine and Biology, vol. 47, pp. 2525, 2002.

[10] H. Yu and G. Wang, "Feldkamp-type VOI Reconstruction from Super-short-scan Cone-beam Data," Medical physics, vol. 31, pp. 1357-1362, 2004. 